\title{
Conical Emission, Pulse Splitting, and X-Wave Parametric Amplification in Nonlinear Dynamics of Ultrashort Light Pulses
}

\author{
Daniele Faccio, ${ }^{1, *}$ Miguel A. Porras, ${ }^{2}$ Audrius Dubietis, ${ }^{3}$ Francesca Bragheri, ${ }^{4}$ Arnaud Couairon, ${ }^{5}$ and Paolo Di Trapani ${ }^{1}$ \\ ${ }^{1}$ CNR-INFM, Department of Physics and Mathematics, University of Insubria, Via Valleggio 11, 22100 Como, Italy \\ ${ }^{2}$ Departamento de Fisíca Aplicada, ETSIM, Universidad Politécnica de Madrid, Rios Rosas 21, 28003 Madrid, Spain \\ ${ }^{3}$ Department of Quantum Electronics, Vilnius University, Sauletekio Avenue 9, 10222 Vilnius, Lithuania \\ ${ }^{4}$ Department of Electronics, University of Pavia, Via Ferrata 1, 27100 Pavia, Italy \\ ${ }^{5}$ Centre de Physique Théorique, CNRS, UMR 7644, 91128 Palaiseau Cedex, France
}

(Received 1 October 2005; published 16 May 2006)

\begin{abstract}
The precise observation of the angle-frequency spectrum of light filaments in water reveals a scenario incompatible with current models of conical emission (CE). Its description in terms of linear X-wave modes leads us to understand filamentation dynamics requiring a phase- and group-matched, Kerr-driven four-wave-mixing process that involves two highly localized pumps and two $\mathrm{X}$ waves. $\mathrm{CE}$ and temporal splitting arise naturally as two manifestations of this process.
\end{abstract}

DOI: 10.1103/PhysRevLett.96.193901

Filamentation of intense light pulses in nonlinear media has attracted much interest ever since the first evidence in the 1960s ([1] and references therein). Owing to the huge intensities reached in the process, several nonlinear phenomena, e.g., multiphoton absorption, plasma formation, saturable nonlinear response, stimulated Raman scattering, etc., occur in addition to the optical Kerr effect. Indeed, the filament regime is enriched by such peculiar phenomena as pulse splitting, self-steepening, shock formation, supercontinuum generation, and conical emission (CE) [2]. In media with normal group velocity dispersion (GVD), no matter if of solid, liquid, or gaseous nature, CE accompanies filamentation, producing radiation at angles that increase with detuning from the carrier frequency [3,4]. In spite of the generality of the process, a clear understanding of the interplay between $\mathrm{CE}$ and filament dynamics is still missing. Only recently, Kolesik et al. proposed an interpretation of filamentation dynamics on the basis of pulse splitting and dynamic nonlinear $X$ waves at the far field [5], in which the double X-like structure observed in simulated angle-frequency spectra arises from the scattering of an incident field at the two main peaks of the split material response wave.

Originally, CE in light filaments was interpreted in terms of the angle-frequency modulation instability (MI) gain pattern of the plane and monochromatic (PM) modes of the nonlinear Schrödinger equation (NSE) [6,7]. Measurements at large angles and detunings from the carrier frequency gave in fact results fairly compatible with this interpretation $[8,9]$. In the present work, owing to the use of a novel imaging spectrograph technique [3], we have been able to observe the CE in the region of small angles and detunings. The results indicate a scenario not compatible with the MI analysis of PM modes. We propose a simple picture in which the modification of the MI results from the parametric amplification of two weak X waves by the strong, highly localized pump. Supporting this interpretation, we derive, from the matching condition among
PACS numbers: 42.65.Tg, 05.45.Yv, 41.20.Jb, 42.65.Jx

the interacting waves, a simple expression [Eq. (4)] that accurately determines the overall CE structure, and also predicts the splitting velocity of the pump wave as a function of the intensity reached at collapse.

The experiment was carried out using a $3 \mathrm{~cm}$-long water cell as a bulk Kerr medium. Beam filamentation was induced by a $f=50 \mathrm{~cm}$ lens placed $48 \mathrm{~cm}$ before the input facet of the cell that focuses the spatially filtered pulses of carrier wavelength $527 \mathrm{~nm}$ delivered by a modelocked, chirped-pulse regeneratively amplified, Nd:glass laser with $10 \mathrm{~Hz}$ repetition rate (Twinkle; Light Conversion, Ltd.). Input pulse energies are adjusted to a few $\mu \mathrm{J}$ by a half-wave plate and a Glan-Taylor polarizer, and the focal diameter and duration are measured to be $100 \mu \mathrm{m}$ and 200 fs (FWHM), which implies focal intensities of the order of $10^{11} \mathrm{~W} / \mathrm{cm}^{2}$. The output facet of the cell was imaged with a 4- $f$ telescope onto the rear focal plane of a second lens $\left(f_{F}=15 \mathrm{~cm}\right)$ used to obtain the spatial Fourier transform of the filament. The input slit of the imaging spectrograph (MS260i, Lot-Oriel) with a high-resolution 1200 lines/mm diffraction grating was placed at a distance $f_{F}$ from the Fourier lens so as to reconstruct the anglewavelength $(\theta-\lambda)$ spectrum of the filament on a commercial CCD 8-bit camera (Canon), placed at the output plane of the spectrograph [3]. We recorded only single-shot spectra in order to avoid averaging effects due to possible shot-to-shot energy fluctuations, and highly saturated the central peak of the spectrum so as to highlight the surrounding structure.

For input energy $E_{\text {in }} \lesssim 1.8 \mu \mathrm{J}$, no CE or clear X-like features were observed. Figure 1 shows a $\theta-\lambda$ spectrum for $E_{\text {in }}=2 \mu \mathrm{J}$, where CE appears as distinctly separated redshifted and blueshifted X-shaped tails. This pattern remains very similar with increasing input energy up to $E_{\text {in }} \sim 4 \mu \mathrm{J}$, while further increase produces a slowly deteriorating picture, with a modulated intensity pattern that extends to nearly all recorded values of $\theta$ and $\lambda$. We interpret this deterioration as due to the onset of local 


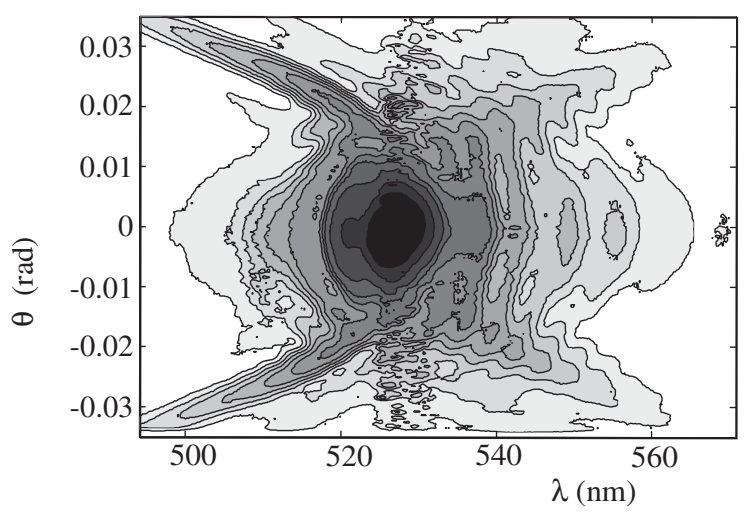

FIG. 1. Contour plot (in logarithmic scale over two decades) of the measured $\theta-\lambda$ spectrum for $E_{\text {in }}=2 \mu \mathrm{J}$.

breakdown in the water sample. Similar X-shaped patterns have been also described at different wavelengths (400 and $800 \mathrm{~nm}$ ) in the normal dispersion region [10,11].

$\mathrm{CE}$ is commonly accepted to arise from the interplay of diffraction, dispersion, and nonlinear response, the simplest theoretical model that accounts for it being the cubic NSE with normal GVD [6,7],

$$
\frac{\partial A}{\partial z}=\frac{i}{2 k_{0}} \nabla_{\perp}^{2} A-\frac{i k_{0}^{\prime \prime}}{2} \frac{\partial^{2} A}{\partial \tau^{2}}+i \frac{\omega_{0} n_{2}}{c}|A|^{2} A .
$$

Here, $A(x, y, \tau, z)$ is the envelope of the wave packet $E=A \exp \left(i k_{0} z-i \omega_{0} t\right)$ of carrier frequency $\omega_{0}, \nabla_{\perp}^{2}=$ $\partial^{2} / \partial x^{2}+\partial^{2} / \partial y^{2}$ the Laplace operator perpendicular to the propagation direction $z, \tau=t-k_{0}^{\prime} z$ the local time, $c$ the speed of light in vacuum, $n_{2}$ the nonlinear refractive index, and $k_{0}^{(m)} \equiv\left[d^{m} k(\omega) / d \omega^{m}\right]_{\omega_{0}}$, where $k(\omega)$ is the propagation constant. The $\theta-\lambda$ spectrum where $\mathrm{CE}$ is observable is directly related to the Fourier spectrum of $A$. If, for instance, $A(r, \tau, z)$ [with $r=\left(x^{2}+y^{2}\right)^{1 / 2}$ ] is an axially symmetric envelope, and $\hat{A}\left(K_{\perp}, \Omega, z\right)$ [with $K_{\perp}=$ $\left(k_{x}^{2}+k_{y}^{2}\right)^{1 / 2}$ ] is its spatiotemporal Fourier transform, then the angle-frequency $(\theta-\Omega)$ spectrum is given by $\left|\hat{A}\left(k_{0} \theta, \Omega, z\right)\right|^{2}$, where $\theta=K_{\perp} / k_{0}$ is a propagation angle with respect to the $z$ axis, and $\Omega$ a detuning from $\omega_{0}$. The allied $\theta-\lambda$ spectrum is related to the $\theta-\Omega$ spectrum through the change $\Omega=2 \pi c / \lambda-\omega_{0}$.

In this frame, an accepted approach for understanding $\mathrm{CE}$ relies upon the evaluation of the MI gain profile of the PM modes of the NSE [6]. In Kerr self-focusing media with normal dispersion, the perturbations to a PM mode that grows at maximum rate are those whose spatiotemporal frequencies $\left(K_{\perp}, \Omega\right)$ are related by

$$
K_{\perp}(\Omega)=\sqrt{k_{0} k_{0}^{\prime \prime} \Omega^{2}+2 k_{0} \tilde{\beta}} \quad(\tilde{\beta}>0),
$$

i.e., a hyperbola on the $K_{\perp}-\Omega$ plane featuring an angle gap ( $K_{\perp}$ gap) [6]. In this respect, Luther et al. proposed an intuitive picture that assumes the largest MI gain to occur at angles and frequencies fulfilling the linear phasematching condition of the four-wave-mixing (FWM) pro- cess supported by the Kerr response [7]. Under this hypothesis, the asymptotic linear approximation $K_{\perp}(\Omega) \simeq$ $\sqrt{k_{0} k_{0}^{\prime \prime}}|\Omega|$ was reobtained, and the observed discrepancies were attributed to the nonlinear phase shift produced by the PM mode on the weak perturbation.

Preceding experimental observations of $\mathrm{CE}$ in borosilicate glass [8] and ethylene glycol [9] were indeed interpreted to present a hyperbolic structure with an angle gap, which was also attributed to the nonlinear phase shift. This trend is also visible in our measurements (Fig. 1) as an intersection of the two $\mathrm{X}$ arms at a nonzero angle. If we attempt to fit Eq. (2) (taking $\tilde{\beta}$ and $k_{0}^{\prime \prime}$ as free parameters) to the experimental data [see Fig. 2(a), black dotted line and solid circles, respectively], we obtain $k_{0}^{\prime \prime}=0.053 \pm$ $0.03 \mathrm{fs}^{2} / \mu \mathrm{m}$, which firmly supports the predictions [7] regarding the asymptotic slope $\left(k_{0}^{\prime \prime}=0.055 \mathrm{fs}^{2} / \mu \mathrm{m}\right.$ at $527 \mathrm{~nm}[12])$; however, the angle-gap fitting strongly departs from experimental data in the nonasymptotic region, which calls for a fitting to a hyperbola with opposite curvature, that is, to a hyperbola with a frequency gap. This observation advocates a novel interpretation of the origin of CE.

To understand our proposal, note first that Eq. (2) represents the dispersion relation of an X-wave mode of the medium [13], i.e., a diffraction- and dispersion-free wave packet due to compensation of cone-angle-induced dispersion $\left[\theta(\Omega) \simeq K_{\perp}(\Omega) / k_{0}\right]$ and material GVD. The dispersion relation (2) belongs to an $\mathrm{X}$ wave of carrier frequency
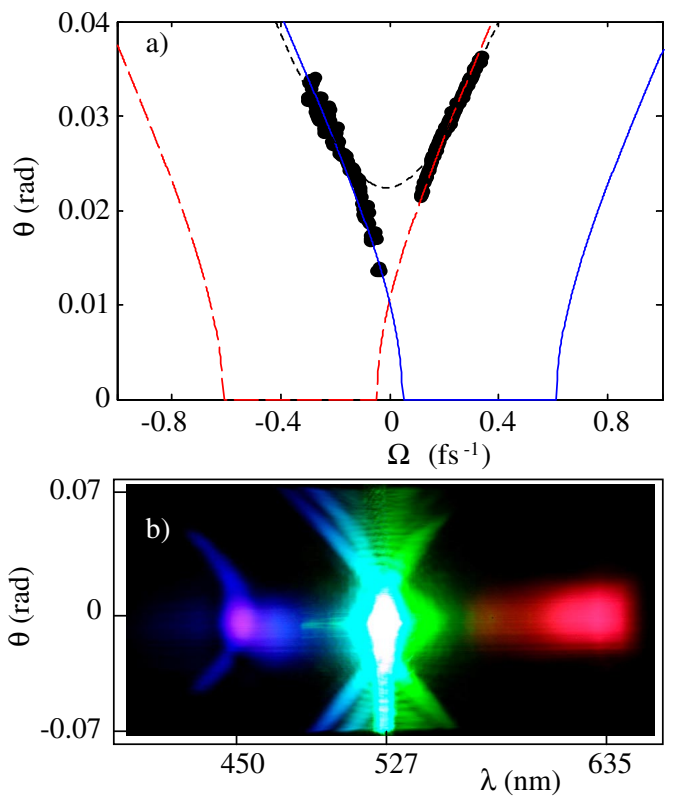

FIG. 2 (color online). (a) Solid circles, $\theta-\Omega$ distribution of peak fluence in Fig. 1; black dotted line, best fit of the entire set of experimental data with Eq. (2); red dashed line, best fit of the $\Omega<0$ data with Eq. (3); blue solid line, best fit of the $\Omega>0$ data with Eq. (3). (b) Experimentally measured $\theta-\lambda$ spectrum: input energy is increased to $E_{\text {in }}=3 \mu \mathrm{J}$ to enhance the visibility of the new tails. 
$\omega_{0}$ and shortened axial wave vector $k_{0}-\tilde{\beta}$. MI may thus be reread in terms of the excitation of a weak, linear $\mathrm{X}$-wave mode by a strong, nonlinear PM pump. If we take into account the nonlinear corrections to the wave vectors of both the pump and $\mathrm{X}$ wave, phase matching reads as $k_{0}+\Delta k=k_{0}-\tilde{\beta}+2 \Delta k$, where $\Delta k=\omega_{0} n_{2} I / c$ is the positive (in self-focusing medium) nonlinear correction to the wave vector of the PM pump of intensity $I$, and $2 \Delta k$ is the corresponding correction for the weak perturbation $[8,9]$, which leads to the positive value $\tilde{\beta}=$ $\omega_{0} n_{2} I / c$, as predicted by the standard MI analysis [6].

We also note that a medium with GVD supports more general $\mathrm{X}$-wave modes with strengthened and shortened wave vectors ( $\tilde{\beta}<0$ and $\tilde{\beta}>0$ ), as well as with blue- and redshifted carriers $\omega_{0}+\tilde{\Omega}$, and general dispersion relation [13]

$$
K_{\perp}(\Omega)=\sqrt{k_{0} k_{0}^{\prime \prime}(\Omega-\tilde{\Omega})^{2}+2 k_{0} \tilde{\beta}} \quad(\tilde{\beta} \gtreqless 0) .
$$

Strictly speaking, $X$ waves in space and time also require phase locking among the spectral components [13]. This assumption is implicit in this work, and is justified by previously measured X-like patterns of filaments in the near field [10]. The phase and group velocities of $\mathrm{X}$ waves are $\boldsymbol{v}^{(p)}=\left(\omega_{0}+\tilde{\Omega}\right) /\left[k\left(\omega_{0}+\tilde{\Omega}\right)-\tilde{\beta}\right]$ and $v^{(g)}=1 / k^{\prime}\left(\omega_{0}+\tilde{\Omega}\right)$, respectively, which can take both subluminal and superluminal values. In practice, Eq. (3) describes a two-parameter family of hyperbolas, sharing the same asymptotic slope $\sqrt{k_{0} k_{0}^{\prime \prime}}$ but admitting angle $(\tilde{\beta}>$ 0 ) and frequency gaps $(\tilde{\beta}<0)$, as well as positive and negative frequency shifts $\tilde{\Omega}$.

The apparent frequency-gap hyperbolic form of the CE, and the actual absence of such a gap, suggests that the CE cannot be described in terms of a single $X$ wave. We then interpret the two observed $X$ tails as belonging to two different $\mathrm{X}$ waves, both featuring frequency gaps, and proceed to fit Eq. (3) with two independent sets of parameters $\tilde{\Omega}$ and $\tilde{\beta}$ (and $k_{0}^{\prime \prime}=0.056 \mathrm{fs}^{2} / \mu \mathrm{m}$ ) to the experimental data. This procedure is also motivated by the X-X structure of the numerically evaluated spectra of filaments in water, which was interpreted to be a consequence of pulse splitting on $\mathrm{X}$-wave generation [5]. Equation (3) with $\tilde{\Omega}=+0.33,-0.33 \mathrm{fs}^{-1}$ and $\tilde{\beta}=-2.2,-2.2 \mathrm{~mm}^{-1}$ (dashed and solid curves, respectively) fits accurately to the experimental data [Fig. 2(a)]. Moreover, this interpretation leads one to suspect the existence of two additional not yet observed $\mathrm{X}$ arms in the spectrum at wavelengths 450 and $635 \mathrm{~nm}$ [Fig. 2(a)]. To verify this prediction, we performed additional measurements with a lower resolution, 300 lines $/ \mathrm{mm}$ diffraction grating in order to cover a wider spectral region [Fig. 2(b)]. Alongside the central tails (around $527 \mathrm{~nm}$ ), we observe the generation of new frequencies in the vicinity of 450 and $635 \mathrm{~nm}$, as expected.

The question then arises of the mechanism responsible for the described spectral structure. We propose that the
$\mathrm{X}-\mathrm{X} \mathrm{CE}$ is a result of a nonlinear FWM interaction in which two weak signal and idler extended $\mathrm{X}$ waves experience a parametric amplification by a strong, highly localized (i.e., non-PM) pump. In order to account for the different extents of the pump and X-wave objects, we took the approximation that self-phase modulation of the pump takes place as for a PM wave, and that no crossphase modulation occurs in the $\mathrm{X}$ waves. In fact, energy does not flow axially in $\mathrm{X}$ waves, but along conical surfaces, which prevents pump-induced phase accumulation to take place.

Under this assumption, phase matching between a localized pump and a single $X$ wave of same frequencies $\omega_{0}$ would require $k_{0}+\Delta k=k_{0}-\tilde{\beta}$, a condition that, contrary to the case of a PM pump, is satisfied by a frequencygap $\mathrm{X}$-wave mode $(\tilde{\beta}=-\Delta k<0)$. We now apply the same reasoning to the more general case of a FWM process involving two degenerate, highly localized pumps and two $\mathrm{X}$ waves. Phase matching is imposed by strict energy and momentum conservation, i.e., $\Omega_{s}=-\tilde{\Omega}_{i}$ and $2 k=k_{s}+$ $k_{i}$, respectively, where $k=k_{0}+\Delta k$ is the nonlinear pump wave number, and $k_{s, i}=k\left(\omega_{0}+\tilde{\Omega}_{s, i}\right)-\tilde{\beta}_{s, i}$ are the two, signal and idler, X-wave numbers. Using $k\left(\omega_{0}+\tilde{\Omega}_{s, i}\right) \cong$ $k_{0}+k_{0}^{\prime} \tilde{\Omega}_{s, i}+k_{0}^{\prime \prime} \tilde{\Omega}_{s, i}^{2} / 2$ the phase-matching constraint leads to the relation $\tilde{\beta}_{s}+\tilde{\beta}_{i}-k_{0}^{\prime \prime} \tilde{\Omega}_{s, i}^{2}=-2 \Delta k$. Among all possible couples of $X$ waves satisfying this relation, those whose spectra cross the pump, located around $\Omega=$ $0, K_{\perp}=0$, are the most energetically favored, since they will not need to grow from noise, but from the more effective pump self-phase modulation. The X-wave-pump crossing condition leads, from Eq. (3), to $\tilde{\beta}_{s, i}=$ $-k_{0}^{\prime \prime} \tilde{\Omega}_{s, i}^{2} / 2$, and then, on account that $\Delta k=\omega_{0} n_{2} I / c$, to

$$
\tilde{\beta}_{s, i}=-\omega_{0} n_{2} I / 2 c .
$$

Accordingly, the two X waves will present symmetrical frequency shifts $\tilde{\Omega}_{i, s}= \pm \sqrt{\omega_{0} n_{2} I / c k_{0}^{\prime \prime}}$, and frequency gaps of width $2\left|\tilde{\Omega}_{i, s}\right|$, from $\Omega=0$ towards the Stokes and anti-Stokes bands, as observed in the experiment.

Equation (4) predicts also a precise dependence of the whole CE structure on the pump intensity $I$, whose validity we have tested numerically. We solved the NSE (1) with cylindrical symmetry by means of a transverse CrankNicholson scheme applied to each frequency component of the pulse [10], and with $k_{0}^{\prime \prime}=0.056 \mathrm{fs}^{2} / \mu \mathrm{m}$, the linear refractive index $n=1.33$ and $n_{2}=1.6 \times 10^{-16} \mathrm{~cm}^{2} / \mathrm{W}$, for an input 200 fs-long, $100 \mu \mathrm{m}$-wide (FWHM) Gaussian wave packet of intensity $0.6 \times 10^{11} \mathrm{~W} / \mathrm{cm}^{2}$. Since CE is seen to develop explosively at the collapse, we identified the pump intensity $I$ with the collapse intensity, i.e., with the highest intensity reached during propagation. In order to attain different values of $I$ without changing the rest of parameters, we added to the second member of the NSE the nonlinear dissipation term $-\beta^{(K)}|A|^{2 K-2} A / 2$, with $K=3$ (three-photon absorption at $527 \mathrm{~nm}$ in water), and perform simulations with $\beta^{(K)}$ ranging from $1.2 \times 10^{-23}$ to 


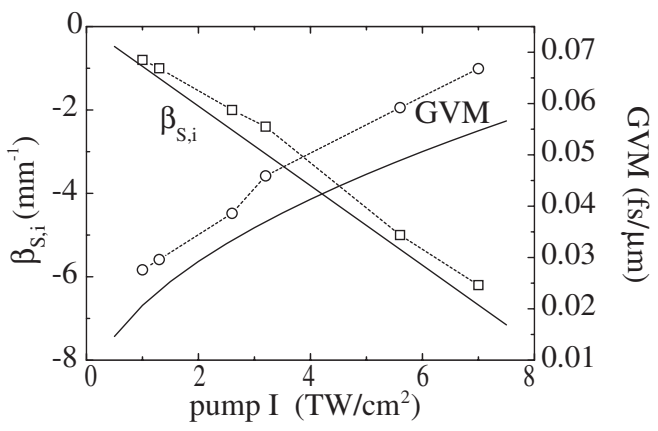

FIG. 3. Left axis: Values of $\tilde{\beta}_{s, i}$ obtained from X-wave fitting to the numerical $K_{\perp}-\Omega$ spectra for increasing pump intensities $I$ (open squares) and prediction of Eq. (4) (solid curve). Right axis: GVM between the splitting pulses obtained from direct space-time numerical simulation (open circles) and between the $\mathrm{X}$ waves as given by $2 \sqrt{k_{0}^{\prime \prime} \omega_{0} n_{2} I / c}$ (solid curve).

$8 \times 10^{-25} \mathrm{~cm}^{3} / \mathrm{W}^{2}$. We neglected self-generated plasma, as we are investigating a regime in which the single filament peak intensity is relatively low (so that any material breakdown effects may also be neglected) [14]. The $K_{\perp}-\Omega$ spectra obtained numerically (not shown) are symmetric with respect to $\Omega=0$ due to the approximations involved in the NSE, and present a nearly invariant X$\mathrm{X}$ structure beyond the collapse distance (in spite of the quickly decreasing peak intensity), with one tail of each $\mathrm{X}$ wave nearly passing through $\Omega=0$, as also observed experimentally. The simulated spectrum at $3 \mathrm{~cm}$ from the input plane was then easily fitted with two $\mathrm{X}$-waves modes crossing $\Omega=0$ [i.e., with $k_{0}^{\prime \prime}=0.056 \mathrm{fs}^{2} / \mu \mathrm{m}$ and $\tilde{\Omega}_{s, i}=$ $\left.\pm\left(-2 \tilde{\beta}_{s, i} / k_{0}^{\prime \prime}\right)^{1 / 2}\right], \tilde{\beta}_{s, i}$ being then the only one free parameter. Figure 3 shows the values of $\tilde{\beta}_{s, i}$ obtained from the best fits to the simulated spectra, versus pump intensity $I$ (open squares). The excellent agreement with the prediction of Eq. (4) (solid line) strongly supports our FWM analysis.

Extending further our interpretation, we conjecture that temporal splitting in filamentation with normal GVD, usually described from a purely temporal perspective, is a consequence of higher-order matching among the interacting waves - in our case, of group matching. Condition (4) entails that the two $\mathrm{X}$ waves travel at different group velocities $v_{s, i}^{(g)}=1 / k^{\prime}\left(\omega_{0}+\tilde{\Omega}_{s, i}\right)=1 /\left[k_{0}^{\prime} \pm k_{0}^{\prime \prime}\left|\tilde{\Omega}_{s, i}\right|\right]$, and therefore split apart with a group velocity mismatch $(\mathrm{GVM}) 1 / v_{s}^{(g)}-1 / v_{i}^{(g)}=2 k_{0}^{\prime \prime}\left|\tilde{\Omega}_{s, i}\right|=2 \sqrt{k_{0}^{\prime \prime} \omega_{0} n_{2} I / c}$ proportional to $\sqrt{I}$. Group matching among the interacting waves is then better attained if the two pumps, breaking their initial degeneracy, split also to copropagate with the $\mathrm{X}$ waves. In this view, pulse splitting is not a mere collapsearresting mechanism [7], which further determines, taking for granted, the generation of two $X$ waves [5]. In contrast, $\mathrm{X}$-wave instability (or parametric amplification) and splitting instability are not independent phenomena, but two aspects of the same process. To support this hypothesis, we have evaluated the GVM of the split pumps in the same numerical simulations as in the preceding paragraph. From plots of the on-axis temporal profiles at different distances beyond collapse, we obtained the pump GVM as the slope of a linear fitting to the time delay between the two peaks versus distance $z$. Figure 3 shows that the pump GVM (open circles) does not depart more than $10 \%$ from the predicted X-wave GVM (solid curve), and that both follow a similar dependence with the intensity $I$ at collapse. Our analytical description has been achieved by use of two approximations: (i) strict energy (and momentum) conservation and (ii) the description of pump and $\mathrm{X}$ waves in terms of their carriers only. The symmetry resulting from this analysis might be violated if higher-order dispersive and nonlinear effects are no longer negligible.

In summary, our experiments demonstrate that $\mathrm{CE}$ in the angular spectrum of filaments is not interpretable in the frame of MI of PM modes of the NSE. Strong localization of the self-focusing pulse substantially modifies the MI pattern, which finds accurate description in terms of linear $\mathrm{X}$-wave modes of the medium, and simple explanation as a result of a dominant, phase-matched FWM mixing process supported by the NSE dynamics between two highly localized, strong pump waves and two amplifying weak $\mathrm{X}$ waves. Pulse temporal splitting emerges in this model as the necessary temporal dynamics for preserving group matching among the interacting waves.

*Electronic address: daniele.faccio@uninsubria.it

[1] J. Marburger, Prog. Quantum Electron. 4, 35 (1975).

[2] A. Gaeta, Science 301, 54 (2003).

[3] D. Faccio, P. Di Trapani, S. Minardi, A. Bramati, F. Bragheri, C. Liberale, V. Degiorgio, A. Dubietis, and A. Matijosius, J. Opt. Soc. Am. B 22, 862 (2005).

[4] E. T. J. Nibbering, P. F. Curley, G. Grillon, B. S. Prade, M. A. Franco, F. Salin, and A. Mysyrowicz, Opt. Lett. 21, 62 (1996).

[5] M. Kolesik, E. M. Wright, and J. V. Moloney, Phys. Rev. Lett. 92, 253901 (2004).

[6] L. W. Liou, X. D. Cao, C. McKinstrie, and G. P. Agrawal, Phys. Rev. A 46, 4202 (1992).

[7] G. G. Luther, A.C. Newell, J. V. Moloney, and E. M. Wright, Opt. Lett. 19, 789 (1994).

[8] R. Alfano and S. Shapiro, Phys. Rev. Lett. 24, 584 (1970).

[9] Q. Xing, K. M. Yoo, and R. R. Alfano, Appl. Opt. 32, 2087 (1993).

[10] D. Faccio, A. Matijosius, A. Dubietis, R. Piskarskas, A. Varanavicius, E. Gaizauskas, A. Piskarskas, A. Couairon, and P. Di Trapani, Phys. Rev. E 72, 037601 (2005).

[11] M. Kolesik, G. Katona, J. V. Moloney, and E. M. Wright, Phys. Rev. Lett. 91, 043905 (2003).

[12] A. Van Engen, S. Diddams, and T. Clement, Appl. Opt. 37, 5679 (1998).

[13] M. A. Porras and P. Di Trapani, Phys. Rev. E 69, 066606 (2004).

[14] M. Geissler et al., Phys. Rev. Lett. 83, 2930 (1999). 